CYBERNETICS AND INFORMATION TECHNOLOGIES • Volume 16, No 6 Special issue with selection of extended papers from 6th International Conference on Logistic, Informatics and Service Science LISS'2016

Sofia $\bullet 2016$

\title{
A Game Theory Based Model for Internet Public Opinion's Embryonic Stage
}

\author{
Chen Chen ${ }^{1}$, Honglu Liu ${ }^{1}$, Xiaolan Guan ${ }^{2}$ \\ ${ }^{1}$ School of Traffic and Transportation, Beijing Jiaotong University, Beijing, China \\ ${ }^{2}$ Beijing Institute of Graphic Communication, Beijing, China \\ Emails:14120961@bjtu.edu.cn hlliu@bjtu.edu.cn 08113101@bjtu.edu.cn
}

\begin{abstract}
The development of internet public opinion presents a certain ecological characteristics. According to the different characteristics, this paper divides internet public opinion into five stages. This paper focuses on the first stage in internet public opinion, from the perspective of qualitative and quantitative analysis, to have a definition of embryonic stage, characteristics, as well as composition. Then this paper analyzes the causes of the formation mechanism in internet public opinion's embryonic stage with the method of game theory. After that, the concepts of hot source factors are introduced to construct the conceptual model of embryonic stage in internet public opinion. Finally, aiming at different types of hot source factors (internet public opinion events), we put forward the effective means to guide and control the development of embryonic stage.
\end{abstract}

Keywords: Internet public opinion, embryonic stage, game theory, formation mechanism, conceptual model.

\section{Introduction}

With the rapid development of microblog and forum the Internet media, the Internet has become an important platform of public opinion. As an important part of the social public opinion, internet public opinion has gradually got people's attention. Having a research on the formation mechanism of the internet public opinion and recognizing the characteristics of embryonic stage is helpful to guide and control the internet public opinion, which is important to study the internet public opinion.

So far, the researches on internet public opinion mainly focus on three aspects. The first aspect is the research on evolution model of internet public opinion, which is representative by the Sznajd model [1], Deffuant model [2], Krause and Hegselmann model [3]. The second aspect is the research on the monitor and alteration of internet public opinion, which is representative by the study of $\mathrm{Zeng}$ and $\mathrm{Hu}$ [4] and $\mathrm{Xu}, \mathrm{Zhang}$ and Li [5]. The third aspect is the research on the development stage of internet public opinion, which is representative by the study 
of $\mathrm{Han}$ and $\mathrm{Huo}$ [6]. In foreign research, K. Ki m, Y. M. B ae k and N. Kim [7] made research on formation of online public opinion; Wojcieszak and Mutz [8] made research on the embryonic and dissemination process of internet public opinion in the complex network. In the existing of research on the development stage of internet public opinion, we can know that most scholars have studied the evolution rules from the perspective of qualitative, and few scholars study the fluctuation and causes of internet public opinion from the perspective of qualitative and quantitative. In addition, most scholars only have a simple analysis of the development process of the whole internet public opinion, and they did not make a deep thinking on a certain development stage of internet public opinion.

For the research on the formation mechanism of the embryonic stage, Noell e-N e u mann [9] has proposed the Spiral theory of silence, which thinks that silence of one side may cause the opinion enhancement of the other side in the formation of the embryonic stage, and promoting social integration objectively in this cycle then ensuring to reach a certain level of agreement in the concepts and behaviors. Bi Hongyin [10, 11] believes that in the process of information exchange in internet public opinion, the existence of the netizens' collective psychology make expression influenced by the community changed and even distorted. Through the study of group pressure, group polarization, collective unconscious, group interaction, etc., he shows the group influence in the internet public opinion formation and changes.

The study of $\mathrm{W}$ ang and $\mathrm{Yu}$ u en [12] has confirmed that the development process of Internet public opinion present a certain ecological characteristics. According to the different properties and characteristics of the events sensitivity, tendency of Internet users, the amount of netizens' participation, communication channels and the focal network posts in the different stages of internet public opinion, internet public opinion is divided the into five stages: embryonic stage, outbreak stage, diffusion stage, transitional stage and decaying stage, as shown in Fig. 1. Because the evolution of internet public opinion is in accord with ecological laws and the game theory is used to study the behaviour of human beings with complete rationality, it is accord with objective law to study internet public opinion with method of the game theory [13].

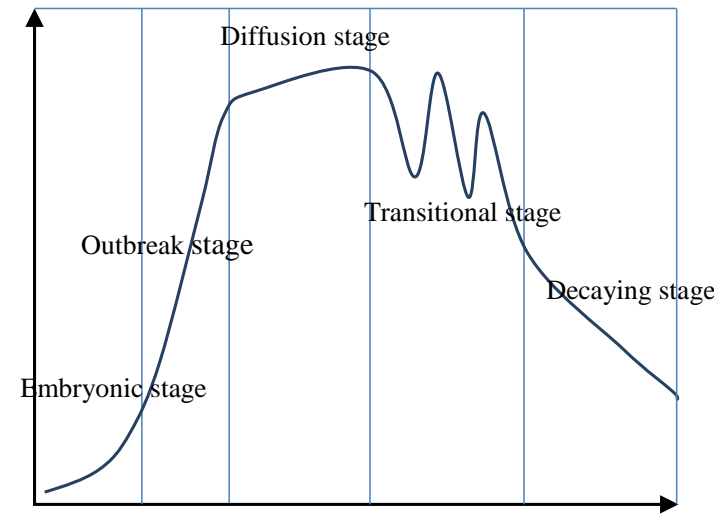

Fig. 1. Evolution process of internet public opinion 


\section{The definition of embryonic stage in internet public opinion}

\subsection{The concept of internet public opinion}

At present, scholars at home and abroad have a variety of definition of internet public opinion. There are some representative opinions: B l u me r Herbert [14] thinks that the internet public opinion is a kind of collective behaviour which is composed by the people who are discussed in the public places. Yi Li u [15] in Tianjin Academy of Social Sciences believes that the internet public opinion is a collection through internet to express and spread a variety of emotions, intentions, attitudes and views. Zhou and Wang [16] from Zhongshan University believes that the Internet public opinion is for a certain "focus", "hot spots" of the event, some influence and tendency of opinion or speech that social public showed up.

In this paper, on the basis of domestic and international existing definitions, we need to clear the following basic characteristics of the internet public opinion:

- The essence of internet public opinion is the same as traditional public opinion. Internet public opinion and traditional public opinion are both the collection of people's attitude, opinions and emotions to some public affairs and real life. But the difference is the internet public opinion spread through the network platform, and the traditional public opinion spread through the traditional media.

- The internet public opinion is the reflection of real public opinion. Internet public opinion often originates from the reality of the public opinion, and it just transfer expression and dissemination places from realistic environment to the Internet.

- There are some differences between the internet public opinion and the real public opinion. Compared to the traditional platform, internet has convenient, identity hidden, difficult to track and status equally characteristics. Therefore, there are certain difference between the study of internet public opinion and traditional public opinion.

\subsection{The description of embryonic stage}

The definition of embryonic stage in internet public opinion is the sensitive information with public opinion events emerging and resulting in the production of related topics. Of course, only when the internet event developed into an internet public opinion, the embryonic stage of internet public opinion dose makes sense. In other words, the embryonic stage is not alone, and it depends on the whole process of internet public opinion development. In the development of internet public opinion, from the perspective of qualitative, we can summarize the characteristics of the internet public opinion in the embryonic stage as shown in Table 1.

In the development stage of internet public opinion, from the quantitative point of view, two variables which are internet public opinion heat and internet public opinion sensitivity are introduced to describe the position of embryonic stage in the whole internet public opinion process.

Internet public opinion heat refers to the upsurge degree of the network media reporting and the netizens' discussion. Internet public opinion heat takes the form of 
the amount of event information specifically, such as the amount of discussion in the We-Media, the amount of reporting on the internet media, and so on.

Table 1. The characteristics of embryonic stage in internet public opinion

\begin{tabular}{|c|c|}
\hline Embryonic stage & Characteristics \\
\hline $\begin{array}{c}\text { The sensitivity of Internet } \\
\text { events }\end{array}$ & $\begin{array}{l}\text { A small part of netizens concern it, and some people are still in } \\
\text { hesitation and wait-and-see condition }\end{array}$ \\
\hline Tendency of netizens & Support, oppose and neutral \\
\hline $\begin{array}{c}\text { Attitude of government and } \\
\text { official media }\end{array}$ & Concern, support, oppose and silence \\
\hline Participation of Netizens & Small amount, the amount of post from less to more \\
\hline $\begin{array}{c}\text { Channels of } \\
\text { communication }\end{array}$ & $\begin{array}{l}\text { The internet public opinion evolves and transforms in different } \\
\text { positions such as forum, blog, instant messaging groups, etc. } \\
\text { instead of fixing in a single position }\end{array}$ \\
\hline The key post & Emergence \\
\hline
\end{tabular}

Internet public opinion sensitivity refers to the amplitude of variation about the amount of internet public opinion information at public opinion hot list in a period of time, which is the increasing speed of internet public opinion heat in a certain time specifically. In a certain period of time, the more increasing of the internet public opinion heat is, the more sensitively the internet public opinion sensitivity is.

From the perspective of internet public opinion heat and internet public opinion sensitivity, Malaysia Airlines loss of communication and Beijing APEC for examples, we think the sum of the amount of internet reporting and the amount of discussion at we-media is equal to the amount of internet public opinion approximately, and we think that the amount of internet public opinion is equal to internet public opinion heat approximately, then we draw the internet public opinion heat pictures of the two events, as shown in Figs 2 and 3.

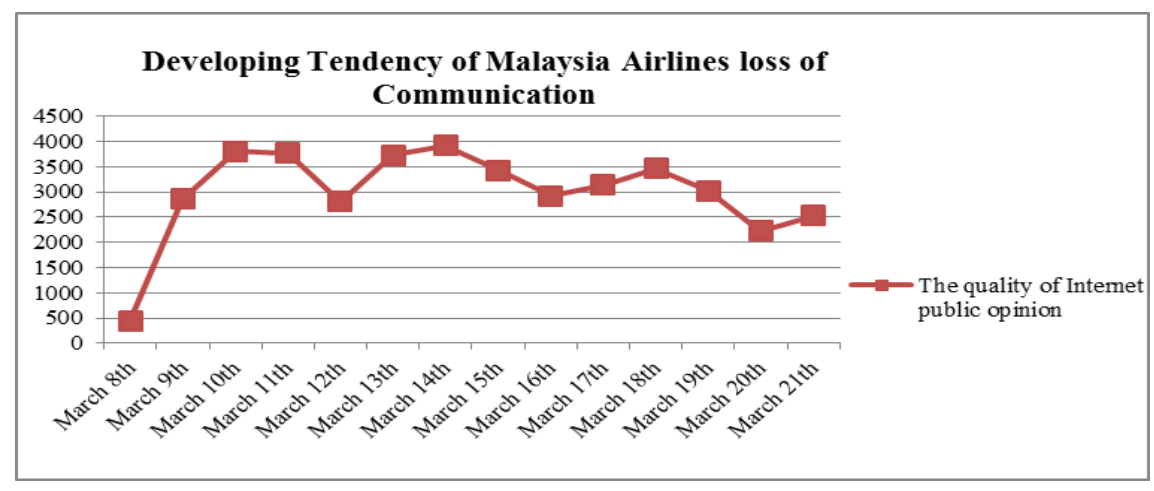

Fig. 2. Developing tendency of Malaysia Airlines loss of communication

From the development process of the Malaysia Airlines loss of communication and Beijing APEC two examples, in the embryonic stage, the Internet public opinion heat is low, which means there is a small quantity of the information about the internet public opinion. From the embryonic stage to the outbreak stage, Internet public opinion sensitivity grows up, which means that there appears a great 
increasing of Internet public opinion heat. Therefore, from the quantitative point of view, the embryonic stage of internet public opinion is from the Internet public opinion heat at 0 to end of the first large numerical of Internet public opinion sensitivity. In this process, the Internet public opinion heat is increasing continual, which means this internet public opinion attracts more people's attention.

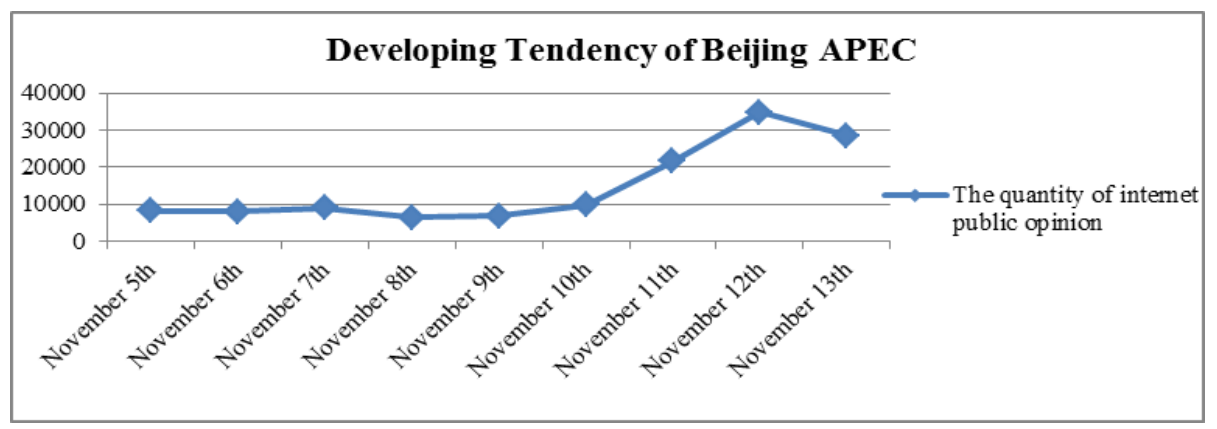

Fig. 3. Developing tendency of Beijing APEC

\subsection{The composition of embryonic stage}

The internet public opinion is the reflection of real public opinion. However, not all the real public opinion or real events are necessary to disseminate in the internet and become internet public opinion. The formation of internet public opinion is based on the three preconditions.

(1) The main body: Internet users with collective interests demanding

Internet users group is the disseminator and affected persons in the internet public opinion. All members of the society have the desire to express their demands, and the anonymity and freedom of internet give netizens more positive social participation consciousness. In real life, due to the existence of some typical social events and social contradictions, which make scattered and netizens with different interests demanding gathering together, and forming an internet users group with collective interests demanding. Obviously, compared to the individual interests demanding, the collective interest demands is more easily to bring the influence power, which may promote social events spread into the internet events and then form the internet public opinion. Therefore, Internet users are the fundamental driving force of internet public opinion's formation.

(2) Object: The issues with representativeness

The formation and development of internet public opinion must rely on some events, and events are often derived from real life. From the Internet public opinion events in recent years, we can be see that those events which can get a high degree concerning often itself has a high degree of sensitivity or has obvious disagreements, such as the events related to the national interests, national economy and the people's livelihood, reflecting the current major social contradictions, and huge disasters, etc.. Therefore, the key to the formation of internet public opinion is the issues with representativeness which can cause widespread concerning. 
(3) Platform: A free internet communication platform

Convenient internet media particularly the We-media provides a relatively freedom environment to the formation of internet public opinion. The relative freedom and anonymity of internet make internet users tend to a critical attitude in expressing emotions and opinions. The virtual and convenient of internet enables users to publish and manufacture information freely, which provides the condition for the internet public opinion. Therefore, a free internet communication platform is the key and guarantee to the internet public opinion formation.

\section{Analysing embryonic stage with the method of game theory}

According to the definition of embryonic stage in internet public opinion, the heat of internet public opinion is the key to the embryonic stage, which means that whether getting into the embryonic stage or not is decided by the amount of netizen's discussion. From the perspective of the whole development process of internet public opinion, only forming a large number of discussions in a short time, the embryonic stage is likely to form, which means the internet public opinion is likely to develop. Definitely, only under the entire internet public opinion dissemination process, the embryonic stage does make sense; otherwise, it means nothing without the whole development process. In the embryonic stage, the behaviour strategies of netizens can be divided into two types: watching and participating. The behaviour strategies netizens used depends on external factors, such as the influence of government, other netizens who have been participated, dissemination platform, etc., which also depends on the internal factors, such as the self-demand of netizens or the event's sensitivity. The internal factors is the essential, the external factors indirectly promote the formation of the embryonic stage through internal factors. Therefore, it can be concluded that the formation mechanism of the embryonic stage in internet public opinion is the result of internal and external factors, as shown in Fig. 4.

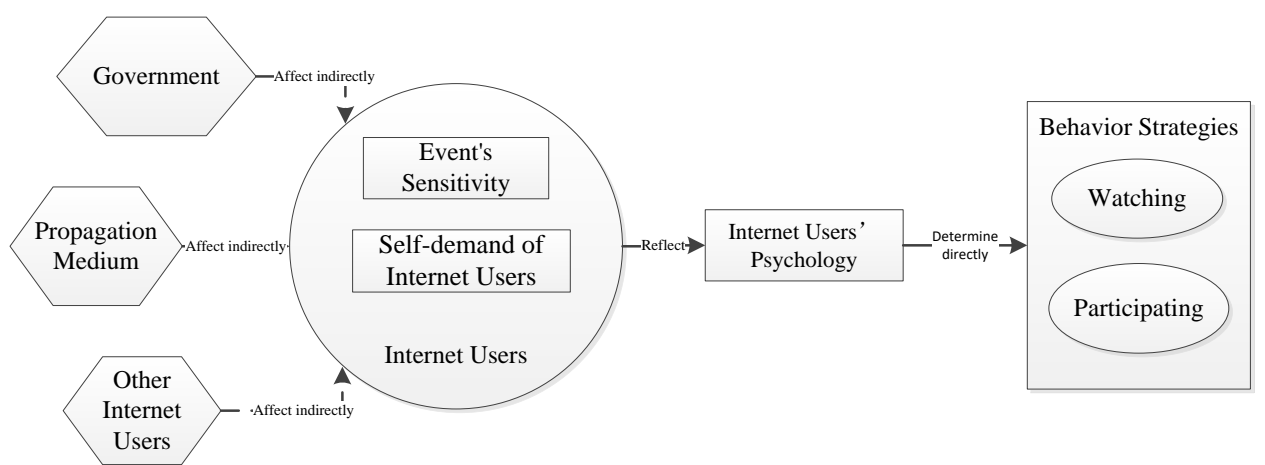

Fig. 4. The formation factors of embryonic stage 


\subsection{Analysis and assumption of model}

The external factors of the embryonic stage contain the effects in the process of the internet public opinion, such as the Internet public opinion dissemination platform, the attitude of the government, the participation of other Internet users, etc. All these factors do not come from the Internet users themselves, but the external factors affect the behaviour of Internet users, which are called the external factors of the embryonic stage. The internet public opinion dissemination platform is a medium of communication, which has no function of information selection and be controlled by the government. Therefore, we assume that the effect made by the Internet public opinion dissemination platform can be ignored. The government's attitude is one of the most important factors in external factors of internet public opinion. We can see that when the attitude of the government is more and more obscure or vaguer, the curiosity and inquiry psychology of netizens are more easily motivated, and netizens are more easily to promote the germination of internet public opinion. In addition, the participation of other internet users is important in external factors of internet public opinion. In the embryonic stage, only a certain number of Internet users participate into the discussion and dissemination, so the internet public opinion gets into the embryonic stage. In order to achieve their own benefit appeals, the netizens who have been participated often persuaded other netizens to participate in the Internet public opinions, which promote internet public opinion to get into the embryonic stage through affecting the amount of participating internet users. In conclusion, the external factors of the embryonic stage can be explained with external model of embryonic stage simply, which mainly are the game theory between government and the Internet users and the effects which are produced by the netizens who have been participated.

This model is based on the following assumptions:

H1: We assumed that the effect produced by the Internet public opinion dissemination platform can be ignored.

$\mathrm{H} 2$ : We assumed that the government has the function of guiding and monitoring the development of internet public opinion.

H3: We assumed that the Internet users give enough concern about the internet public opinion with large risks, wide range of radiation and great influence.

H4: We assumed that internet users will pay less attention to some internet public opinion when the government takes an open and transparent approach to solve some events with lese effects.

H5: We assumed that internet users will pay more attention to some internet public opinion when the government takes an evasive and ambiguous approach to solve some events with the sensitive messages, which is more likely to arouse the embryonic stage and make the internet public opinion develop rapidly.

H6: We assumed that the cost of internet communication can be neglected.

\subsection{Model establishment}

In the external factors model of embryonic stage, it exists an internet public opinion $X$ in a certain range of network space $G$. Internet users $C$ have the need to express their demands. We define the internet users who have been involved in internet 
public opinion discussion as $B$. In the embryonic stage, internet public event $X$ exists, Internet users can take the strategy set $\mathrm{SS}=\{M, N\}, M$ means spread and $N$ means no spread. For the condition $M$, it is divided into support and opposition two forms. So the strategy set $\mathrm{SS}=$ \{support, oppose, watching $\}$, which is expressed by $1,-1,0$. The three strategies can be transformed partly, as shown in Fig. 5.

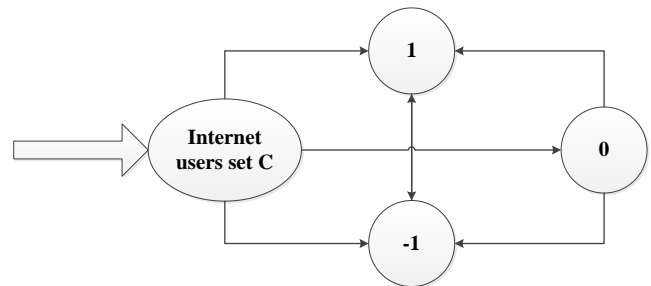

Fig. 5. Internet users $C$ 's strategy transformation in embryonic stage

As the mighty body in the society, government needs to play a key role in guiding and controlling the internet public opinion in the embryonic stage. For the internet public opinion event $X$, government takes the strategy set $\mathrm{SS}^{\prime}=\{C, D\}, C$ means open and transparent treatment of government, and $D$ means evasive and ambiguous treatment of government. Therefore, the strategies between the government and Internet users in the embryonic stage can be divided into the following four circumstances:

In the embryonic stage of internet public opinion, when the government takes an open and transparent approach to internet public opinion event $X$ (treatment $C$ ), then some events with lese effects may not generate a large-scale discussion and dissemination, which means that the internet users will take the watching strategy (strategy $N$ ). The internet public opinion event $X$ failed to get into the embryonic stage, but the potential damage $A$ caused by the government still exist. In this circumstance, the benefit obtained by the internet user is 0 . The benefit obtained by the government is $V-A, V$ means the potential benefits with not getting into the potential benefits, such as the benefit made by some events which may promote the internet public opinion but not promote the public opinion in actual.

There are a number of internet events, even if the government takes the open and transparent treatment (treatment $C$ ), the internet users will still choose to participate into the event (strategy $M$ ) because of the large harmfulness and typical enough of the events, etc., as well as the effect made by other internet users who have been participated. In this circumstance, the loss of government is $\alpha A$, which is denoted by $-\alpha A ; \alpha$ represents diffusivity to the internet users when the government take the open and transparent treatment, which reflect the speed of the dissemination of internet public opinion events. Obviously, $\alpha$ is the dependent variable of the number of internet users $n$ who have been participated, attribute of the internet public opinion $q$ and time $t$, which can be represent by $\alpha=k_{1} f(n, q, t)$. Because the internet users who have been participated the internet public opinion achieve their own benefit appeals, they can get the benefit $P$.

In the embryonic stage of internet public opinion, for the internet public opinion event $X$ with the large harmfulness or influence social stability, the 
government is necessary to take the shielding or evasive treatment (treatment $D$ ). In this circumstance, internet users cannot get access to the event from the internet, and the internet public opinion may not get into the embryonic stage, which means Internet users takes the measure of watching (strategy $N$ ). In this circumstance, the benefit of government is $V$, the loss of internet users is $-L$ because of none the wiser, such as hidden security risks may cause the loss of health of Internet users.

However, not all the events which government takes a shielding or evasive treatment won't get into the embryonic stage of internet public opinion. On the contrary, when government take the shielding or evasive treatment (treatment $D$ ), once the internet users get the event information from other channels such as real space or by word of mouth, etc., it usually leads to internet users producing more intense response to the events, accelerating germination, getting into the embryonic stage faster, and expanding the dissemination areas, although it will take some dissemination cost form those channels because of they are not convenient like internet. In this circumstance, the loss of government is $-\beta A$, in which $\beta$ represents diffusivity to the internet users when the government take a shielding or evasive treatment, which reflect the speed of the dissemination of internet public opinion events. Compared to the open and transparent strategy, a shielding or evasive treatment strategy can more easily aroused netizens to disseminate event, then the spread of event is faster, which means $\alpha<\beta ; \beta$ is also the dependent variable of the number of internet users $\mathrm{n}$ who have been participated, attribute of the internet public opinion $q$ and time $t$, which can be represent by $\beta=k_{2} f(n, q, t)$. In this circumstance, the loss of the internet users is recorded as $-L-C$, and $C$ means the dissemination cost from other channels except internet.

According to the above analysis, we can get the game theory matrix of strategy between government and netizens, as shown in Table 2.

Table 2. Game theory matrix of strategy between government and netizens

\begin{tabular}{|c|c|c|}
\hline \multirow{2}{*}{ Government } & \multicolumn{2}{|c|}{ Netizens } \\
\cline { 2 - 3 } & Spread $(M)$ & No spread $(N)$ \\
\hline Open and transparent treatment $(C)$ & $-\alpha A, P$ & $V-A, 0$ \\
\hline Evasive and ambiguous treatment $(D)$ & $-\beta A,-L-C$ & $V,-L$ \\
\hline
\end{tabular}

3.3. Analysis of balanced development in the embryonic stage

In the embryonic stage, we assumed that the probability of government take the open and transparent treatment (treatment $C$ ) is $x$, and the probability of government take the shielding or evasive treatment (treatment $D$ ) is $1-x$. We assumed that the probability of internet users takes the measure of watching (strategy $N$ ) is $y$, and the probability of internet users takes the measure of participating (strategy $M$ ) is $1-y$.

The benefit of government taking the open and transparent treatment (treatment $C$ ) is

$$
f_{C}=y(V-A)+(1-y)(-\alpha A) .
$$

The benefit of government taking the shielding or evasive treatment (treatment $D$ ) is

$$
f_{D}=y V+(1-y)(-\beta A) .
$$


The average benefit of government is

$$
f_{\text {gov }}=x[(V-A+\alpha A) y-\alpha A]+(1-x)[(V+\beta A) y-\beta A] .
$$

According to the dynamic equation of the evolution game theory, we can get the dynamic evolution equation of the government taking the shielding or evasive treatment (treatment $D$ ):

$$
\begin{gathered}
d x / d t=x\left[f_{\mathrm{gov}}-f_{C}\right]=x\left[x f_{C}+(1-x) f_{D}-f_{C}\right]= \\
=x(x-1)[y(\alpha A-\beta A-A)+(\beta A-\alpha A)] .
\end{gathered}
$$

The benefit of internet users taking the measure of watching (strategy $N$ ) is

$$
f_{N}=x * 0+(1-x)(-M) \text {. }
$$

The benefit of internet users taking the measure of participating (strategy $M$ ) is

The average benefit of internet users is

$$
f_{M}=x P+(1-x)(-M-C) \text {. }
$$

$$
f_{\text {netizens }}=y[0+(1-x)(-M)]+(1-y)[x P+(1-x)(-M-C)] .
$$

According to the dynamic equation of the evolution game theory, we can get the dynamic evolution equation of the internet users taking the measure of watching $($ strategy $N)$ :

$$
\begin{gathered}
d y / d t=y\left[f_{N}-f_{\text {netizens }}\right]=y\left\{f_{N}-\left[y f_{N}+(1-y) f_{M}\right]\right\}= \\
=y(1-y)[C-(C+P) x] .
\end{gathered}
$$

According to differential equation, we can see that $(1,0),(0,1)$ are the equilibrium point of the system, $(1,1)$ and $(0,0)$ are the unstable point, and the $(C /(C+P),(\beta-\alpha) /(\beta-\alpha+1))$ is the saddle point. Then the dynamic evolution of the system is shown in Fig. 6.

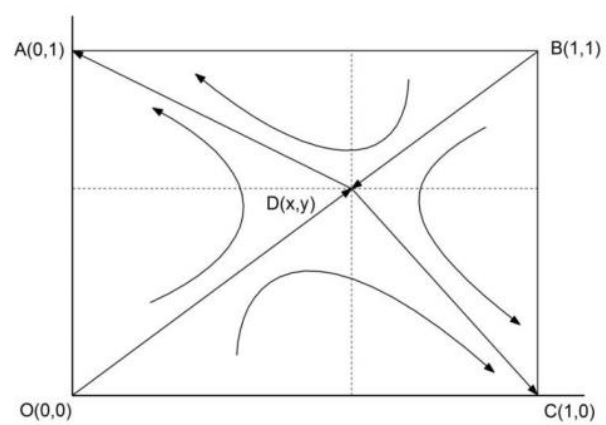

Fig. 6. The dynamic evolution of game theory in the embryonic stage

When $d y / d t=0$, then $x^{*}=C /(P+C)$, in this situation, the $Y$ is the stable state. When $x<x^{*}, y^{*}=1$ is the stable point. When $x>x^{*}, y^{*}=0$ is the stable point. We can see that when the dissemination cost $C$ increased or the benefit of internet users $P$ decreased, the probability of $x<x^{*}$ will be increased when $x^{*}$ is getting increased, the system tends to $y^{*}=1$, which means when the dissemination cost $C$ increased or the benefit of internet users $P$ decreased, internet users are likely to take the measure of watching (strategy $N$ ), the possibility of getting into the embryonic stage is small. Otherwise, when the dissemination cost $C$ decreased or 
the benefit of internet users $P$ increased, the probability of $x>x^{*}$ is increasing, the system tends to $y^{*}=0$, which means when the dissemination cost $C$ decreased or the benefit of internet users $P$ increased, internet users are likely to take the measure of participating (strategy $M$ ), it's likely to get into the embryonic stage.

When $d x / d t=0$, then $y^{*}=(\beta-\alpha) /(\beta-\alpha+1)$, in this situation, the $x$ is the stable state. When $y<y^{*}, x^{*}=1$ is the stable point. And when $y>y^{*}, x^{*}=0$ is the stable point. The measures of government taking depends on the strategy of internet users $y, y$ depends on the speed of the dissemination of internet public opinion $\alpha$ and $\beta, \alpha=k_{1} f(n, q, t), \beta=k_{2} f(n, q, t) ; \alpha$ and $\beta$ are both the increasing function of the number of internet users $\mathrm{n}$ who have been participated, attribute of the internet public opinion $\mathrm{q}$ and time $\mathrm{t}$, and we can know that $k_{2}>k_{1}$ from the above analysis. For an internet public opinion $q, \beta-\alpha$ is growing with the increasing of the number of internet users $\mathrm{n}$ who have been participated and time $t$, and the possibility of $y<y^{*}$ is increasing when $y^{*}$ is growing, the system tend to $x^{*}=1$. In which we can see, for a certain internet public opinion $q$, with the increasing of the number of internet users $n$ who have been participated and time $t$, internet users take the measure of participating (strategy $M$ ), in this time the government should take the open and transparent treatment (treatment $C$ ). Conversely, when the number of internet users $n$ who have been participated and time $t$ are small, the difference of $\beta-\alpha$ is small, system tends to $x^{*}=0$. We can see that, for a certain internet public opinion $q$, when only a few Internet users participate to disseminate the information instead of a large amount of information dissemination, the government can take the shielding or evasive treatment (treatment $D$ ).

\section{The conceptual model of embryonic stage in internet public opinion}

\subsection{Hot source factors of embryonic stage in internet public opinion}

The process of internet public opinion is the result of internal factors and external factors. The reason is complex and diverse. Last chapter has analysed the specific reasons of embryonic stage's formation. This chapter will extract and purify the reasons and construct the conceptual model of embryonic stage. This chapter introduces the concept of hot source factors, and we define hot source factors means an abstract power to make the event's heat rise and keep the heat sustained, which is a collection of internal factors and external factors such as the psychology of netizen, the environment of internet public opinion and the government, etc., which is the direct composition of embryonic stage's formation.

In accordance with content, quality and attribute in the embryonic stage, we can divide the hot source factors of embryonic stage into five categories: multivariate conflict factor, status difference factor, tremendous harmfulness factor, unpredictable factor and misconduct in answer factor.

Multivariate conflict factor refers to the conflict of interests, values and ethics, etc., which can affect the formation of embryonic stage. The factor is the root of the formation of embryonic stage, and almost all the formation of internet public opinion is basically the reflection of different conflicts. This factor is mainly related 
to the national economy and the people's livelihood event. In the background of social transformation, as a member of the social members, internet users are always easy to arouse their self-realization psychology, equal participation psychology and decompression and catharsis psychology when there are conflicts which closely related to their interests, values and ethics, etc.

Status difference factor refers to the disparity in capabilities, roles and positions among the appellor, defendant and involved people in the embryonic stage, and then it causes the unequal status phenomenon. It usually is: the appellor is in a weak position (such as ordinary urban residents, employees, migrant workers), which may have low influence, may have greater pressure to pay the fiddler, may not have a smooth channel to appeal, communicate or vent, may have a deeper concern about their own right, may generate more doubt about the fairness in the profit distribution. Relatively, defendant and involved people are in a strong position in the roles, position, public power, action and influence. Due to the gap between the appellor and defendant, the weakness of appellor is likely to arouse people's sympathy psychology, and it's easy to get into the embryonic stage, which is easy to arouse the netizen's curiosity and inquiry psychology and following suit and conformity psychology.

Tremendous harmfulness factor refers to the result of the internet public opinion event with ecumenical harmfulness, even serious harmfulness or the existence of large potential harmfulness. Because the security issues may involve and threaten everyone, people may have a higher degree of concern about the negative factors that may affect the safety, which is actually deep concern about self-safety and public safety of living environment. Tremendous harmfulness factor is mainly related to the serious natural disaster and some events related to national interests and state interests, which is easy to arouse the netizen's self-realization psychology, curiosity and inquiry psychology and equal participation psychology, then make the internet public opinion getting into the embryonic stage.

Unpredictable factor refers to suddenness, complexity, unpredictable, whirling and confusing of context of the event. The complicated plot makes internet users generate uncertainty when they judge the event and have a social cognition, which may stimulate the internet users to explore the truth with a greater desire. The attribute of unpredictable and unknown may arouse the netizen's curiosity and inquiry psychology, entertainment and fashion psychology, highlighting personality psychology and following suit and conformity psychology, which is mainly related to the entertainment public events or national economy and the people's livelihood event.

Misconduct in answer factor mainly refers to the problems or serious misconduct which is generated in the government or functional departments dealing with internet public opinion, such as government failing to stop the spread of the situation timely and effectively, failing to restore social order and public safety in time, the methods not scientific enough, or omission to the internet public opinion. Misconduct in answer factor is easy to make netizens generate anger and resentment, which may also arouse the netizen's curiosity and inquiry psychology and equal participation psychology easily. Misconduct in answer factor is related to 
the events which can reflect the main social contradictions. In some events, although the internet public opinion is getting into the decaying stage, because of the misconduct or unreasonable of government, the events develop into the embryonic stage again, and enter the public consciousness. Therefore, Misconduct in answer factor is an important factor to intensify and aggravate the internet public opinion.

\subsection{The conceptual model of embryonic stage}

Hot source factors are the direct reasons of the internetwork public opinion's embryonic stage, which are the result of numerous factors' interaction, which is an abstract factor. The internal factor of embryonic stage is an important part of hot source factors, which directly pushes forward the internet public opinion into the embryonic stage. The external factor of embryonic stage is also an important part of hot source factors, which indirectly pushes forward the internet public opinion into the embryonic stage. Therefore, based on the analysis of formation in the embryonic stage and the definition of the hot source factor, we construct the conceptual model of embryonic stage, as shown in Fig. 7.

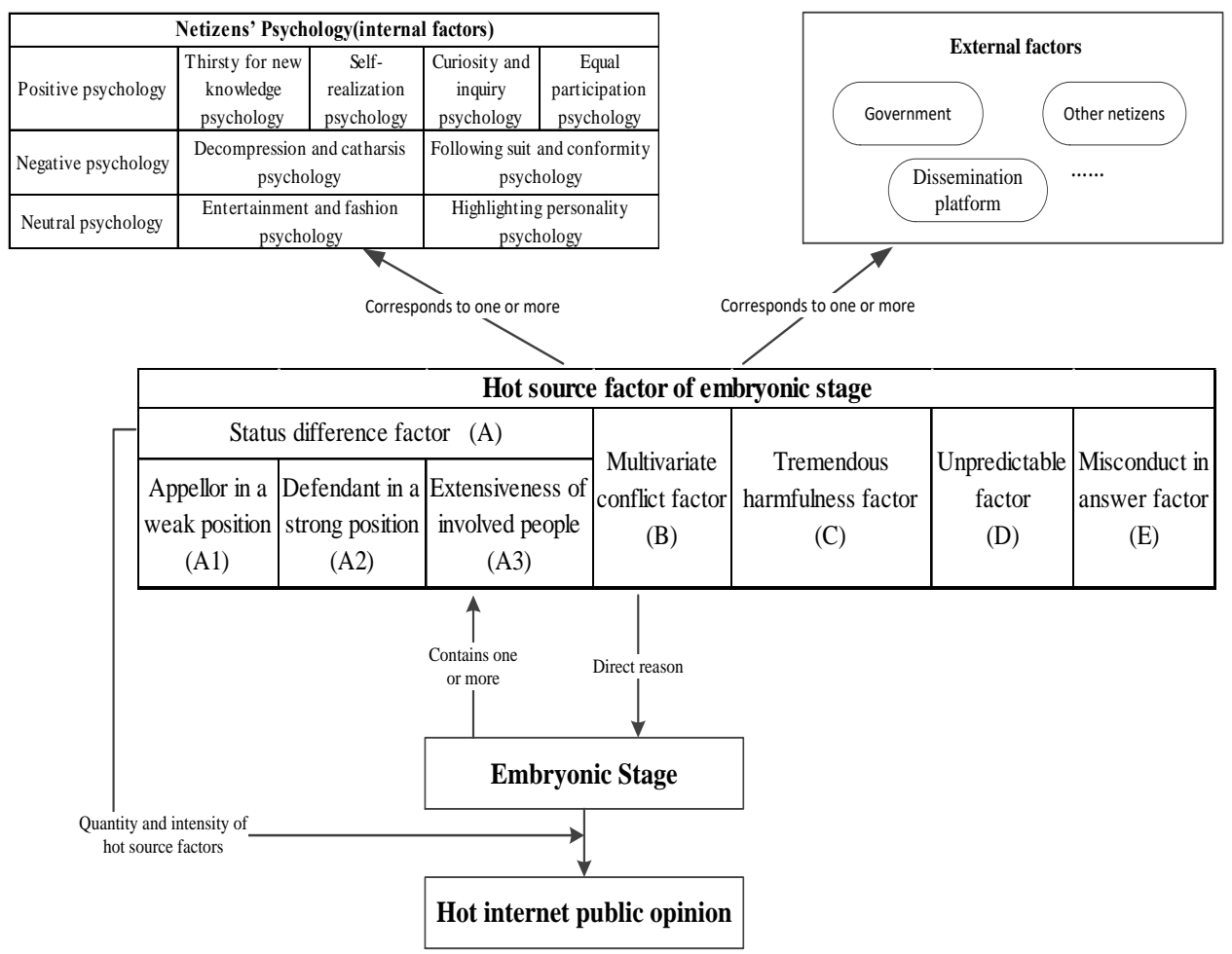

Fig. 7. The conceptual model of embryonic stage

In the conceptual model of embryonic stage, the embryonic stage, the hot source factor, the external factor, the internal factor (psychology of internet users), the relationship of those four has the following characteristics: 
(1) The hot source factors which can reflect the attribute of events usually correspond to one or more internal factors and external factors. The hot source factors are the result of internal factors and external factors to make event get into the embryonic stage.

(2) Embryonic stage of internet public opinion may not necessarily contain all of the hot source factors, but in general, it should contain a heat source factor at least.

(3) There is no direct correlation between the number of hot source factors and whether getting into the embryonic stage. But in general, under the interaction of the different hot source factors, the event containing more hot source factors is more likely to becoming the hot internet public opinion.

(4) Whether the event getting into the embryonic stage and the speed of getting into the embryonic stage is related to the intensity of the hot source factors.

\subsection{Case study}

In this section, we explain the conceptual model of embryonic stage with the case of 2014 global hot internet public opinion-Malaysia Airlines loss of communication.

Morning 2:40 on March 8 2014, the Boeing 777-200 aircraft of Malaysia airlines lost contact with the control center. A Malaysian airplane (MH370) with 227 passengers including 2 children and 12 crews, which included 154 Chinese passengers and this plane, was originally from the Kuala Lumpur to Beijing. This news has caused great concern of public opinion. Since then, from flight location to passport mystery, from terrorist attack to family reaction, although all kind of messages came in a throng, the authority is always a miser with words. So it caused the heat debate of netizens and huge amount of media reports, then it formed internet public opinion.

Compared to other events, Malaysia Airlines loss of communication has distinguishing feature, the existence of disparity in roles and positions between appellor, which is passengers, and defendant, which is Malaysia airlines, the existence of conflict of interests such as life and materials, the characteristic of suddenness and unpredictability, serious harmfulness even related to the safety of life, and evasive and ambiguous treatment of government, etc.. In terms of internal factors of embryonic stage, this event is complicated and confusing, which may easily arouse the netizen's thirsty for new knowledge psychology, self-realization psychology, curiosity and inquiry psychology and equal participation psychology, even some internet users' following suit and conformity psychology. In terms of external factors of embryonic stage, the evasive and ambiguous treatment of government arouses internet users following with their interests. Therefore, Malaysia Airlines loss of communication is related to five hot source factors, which are multivariate conflict factor, status difference factor, tremendous harmfulness factor, unpredictable factor and misconduct in answer factor. Because it contains a number of hot source factors and multiple factors have strong intensity, then resulting in getting into the embryonic stage rapidly and arousing the concern of internet users. 


\section{Summary}

Through the analysis and summary of a large number of hot internet public opinion events, we can know that internet public opinion events, especially hot internet public opinion events are often the result of interaction and intensity of the hot source factors.

The multivariate conflict factor in the embryonic stage of internet public opinion is an aggregation of conflicts, which has the characteristics of long-term accumulation, deep conflict root and great influence. Therefore, the answer and response of multivariate conflict factor is a process of long-term, involving many departments, the national level. In this process, from a macroscopical perspective, stability and unity of the country, fairness and justice of society and people's wellbeing are the essential to answer this factor. From a specific perspective, there are some important measures to answer this factor, such as establishing relevant policies which can narrow the gap between the rich and the poor, giving more support to the disadvantaged group, improving the laws and regulations, policies, rules, regulations, formulation and making them more transparent, strengthening people's participation in government and political affairs, strengthening the supervision of the Internet, etc.

Considering the status difference factor in the embryonic stage of internet public opinion, in the internet public opinion and real public opinion, there are some inequitable judgements which are affected by the gap in the aspects of the role, capacity and condition between the appellor and defendant. But what we need to face up is that the status difference factor internet public opinion is often due to some netizen' subjective assumptions and following suit and conformity psychology, making some fair judgement event in which there are gaps between the appellor and defendant getting super concern and getting into the embryonic stage. This phenomenon makes some internet public opinion just with the characteristic of status difference become the hot event and get netizen's concern, which may affect more Internet users' thinking inertia and cause netizens' subjective impression of some "unfair", then forming a vicious circle. Therefore, the main way to deal with this status difference factor is to strengthen the transparency in the process of law enforcement, to strengthen legal aid for disadvantage groups, to promote positive effect and propagate positive energy, to punish some inequitable national staff sternly, to play a positive leadership role for the law enforcement officers, to monitor the hot events and hot public opinion actively and weaken or transfer the attention of Internet users to status difference factor internet public opinion timely.

The tremendous harmfulness factor in the embryonic stage of internet public opinion is mainly related to the serious natural disaster and some events related to national interests and state interests, and those events have the a sudden and threatening nature, which require the government respond to the public opinion events quickly. The tremendous harmfulness factor internet public opinion has a certain threat and may have a long processing or have the possibility of relapsing, which may cause panic among Internet users. The tremendous harmfulness factor internet public opinion is usually easy to get into the embryonic stage, to last long 
time, and to have another embryonic stage after decaying stage. Therefore, the main way to deal with the tremendous harmfulness factor is: what the government need to do is setting up relevant departments, accumulating the experience, giving the positive and timely response, keeping the process transparently, strengthening the communication of related public opinion events through the internet, preventing the recurrence of embryonic stage, and so on.

The unpredictable factor in the embryonic stage of internet public opinion is mainly related to the entertainment public events or national economy and the people's livelihood event. The internet public opinion event with unpredictable factor has the characteristics of suddenness and plot complexity, which make the event itself relatively complicated and confusing. Added to help intensify the strength of billows and waves of some network promoter and opinion leader, the internet public opinion event with unpredictable factor is easy to get into the embryonic stage. But this factor is usually little associated with the ordinary internet users themselves, so the time of this factor is usually short. When we face with internet public opinion event with unpredictable factor, we can strengthen the supervision of the relevant opinion leaders, guide and transfer the attention of Internet users timely.

The reason for the misconduct in answer factor in the embryonic stage of internet public opinion is clear, and the demanding of internet users is clear. So the coping approach is relatively simple and effective. The main way of dealing with the misconduct in answer factor is: for an internet public opinion event, government should respond to it actively, do not evade, do not retreat, make the information dissemination process transparent, and set up a special institution to answer and monitor the internet public opinion. Besides that, the government also needs to strengthen the positive publicity in daily life and strengthen legislation.

The embryonic stage of internet public opinion is the result of interaction of various factors, which is the result of multiple hot source factors. We need to clear the categories of common hot source factors, and suit the remedy to the case according to the different internet events, different public opinion environment and different netizens' psychology, which is of great significance to guide and control the internet public opinion more effectively.

\section{References}

1. S z n a j d-Wer o n, K., J. S z n a jd. Opinion Evolution in Closed Community. - International Journal of Modern Physics C, Vol. 6, 2000, pp. 1157-1165.

2. Deffuant, G., D. Nea, F. Amblard et al. Mixing Beliefs Among Interacting Agents. Advances in Complex Systems, Vol. 3, 2001, pp. 87-98.

3. H e g s e $1 \mathrm{~m}$ a n n, R., U. Kr a u s e. Opinion Dynamics Bounded Confidence Models, Analysis and Simulation. - Journal of Artificial Societies and Social Simulation, Vol. 3, 2002, pp. 1-33.

4. Z e n g, R., X. X u. Index System and Mechanism of Internet Public Opinion Emergency Warning System. - Journal of Intelligence, Vol. 28, 2009, No 11, pp. 52-54.

5. X u, X., C. Zh ang, W. Li. Retrospect and Prospect of Domestic Research on Network Public Opinion. - Intelligence Theory and the Practice, Vol. 32, 2009, No 3, pp. 115-120. 
6. H a n, L., J. H u o. "Butterfly Effect" and the Network Public Opinion Generation Mechanism. Modern Communication, Vol. 6, 2008, pp. 64-67.

7. K i m, K., Y. M. B a e k, N. K i m. Online News Diffusion Dynamics and Public Opinion Formation: A Case Study of the Controversy over Judges, Personal Opinion Expression on SNS in Korea. - The Social Science Journal, Vol. 2, 2015.

8. Wojcieszak, M. E., D. C. Mutz. Online Groups and Political Dis-Course: Do Online Discussion Spaces Facilitate Exposure to Political Disagreement? - Journal of Communication, Vol. 1, 2009, pp. 40-56.

9. No e 11 e-N e u man n, E. E. The Spiral of Silence. Public Opinion - Our Social Skin. Second Edition. Chicago, University Chicago Press, 1993, pp. 50-56.

10. B i, H. The Analysis of the Netizens' Psychological Characteristics. - Social Sciences Review, Vol. 9, 2006, pp. 38-39.

11. B i, H. Ten Years the Review and Reflection of Modern Public Opinion Research. - Journal of Tianjin Academy of Social Sciences, Vol. 4, 2013, No 4, pp. 67-71.

12. Wang, J., S. Yuwen. Structure and Operation Mechanism of Network Public Opinion Ecosystem Research. - Journal of Intelligence Theory and the Practice, Vol. 37, 2014, No 1, pp. 55-58.

13. Wa n g, Y., K. Yo u, M. Wa n g. Network Public Opinion Propagation Model Based on Game Theory. - Journal of Research in Computer Application, Vol. 30, 2013, No 8, pp. 2480-2482.

14. B lu mer, H. Public Opinion and Public Opinion Polling. - American Sociological Review, Vol. 13, 1947, No 5, pp. 542-549.

15. L i u, Y. Introduction to Network Public Opinion Research. Tianjin, Tianjin People's Press, 2007, pp. 53-55.

16. Z h o u, R., T. W a n g. Internet Public Opinion: The New Field of Modern Ideological and Political Education. - Journal of Ideological and Theoretical Education, Vol. 11, 2005, pp. 12-15. 